\title{
Time-domain and energetic bombardment effects on the nucleation and coalescence of thin metal films on amorphous substrates
}

Daniel Magnfält, Viktor Elofsson, G Abadias, Ulf Helmersson and Kostas Sarakinos

\section{Linköping University Post Print}

\section{Tweet}

N.B.: When citing this work, cite the original article.

Original Publication:

Daniel Magnfält, Viktor Elofsson, G Abadias, Ulf Helmersson and Kostas Sarakinos, Timedomain and energetic bombardment effects on the nucleation and coalescence of thin metal films on amorphous substrates, 2013, Journal of Physics D: Applied Physics, (46), 21. http://dx.doi.org/10.1088/0022-3727/46/21/215303

Copyright: Institute of Physics: Hybrid Open Access http://www.iop.org/

Postprint available at: Linköping University Electronic Press http://urn.kb.se/resolve?urn=urn:nbn:se:liu:diva-95508 


\title{
Time-domain and energetic bombardment effects on the nucleation and coalescence of thin metal films on amorphous substrates
}

\author{
D Magnfält ${ }^{1,3}$, V Elofsson ${ }^{1}, \mathrm{G}$ Abadias $^{2}, \mathrm{U}_{\text {Helmersson }}{ }^{1}$, and K Sarakinos ${ }^{1}$ \\ ${ }^{1}$ Plasma and Coatings Physics Division, Department of Physics, Chemistry and Biology (IFM), \\ The Institute of Technology, Linköping University, SE-581 83 Linköping, Sweden \\ 2 Institut P', Département Physique et Mécanique des Matériaux, Université de Poitiers-CNRS- \\ ENSMA, SP2MI, Téléport 2, Bd M. et P. Curie, F-86962 Chasseneuil-Futuroscope, France \\ e-mail: danma@ifm.liu.se
}

\begin{abstract}
Pulsed, ionized vapor fluxes, generated from high power impulse magnetron sputtering (HiPIMS) discharges, are employed to study the effects of time-domain and energetic bombardment on the nucleation and coalescence characteristics during Volmer-Weber growth of metal (Ag) films on amorphous $\left(\mathrm{SiO}_{2}\right)$ substrates. In situ monitoring of the film growth, by means of wafer curvature measurements and spectroscopic ellipsometry, is used to determine the film thickness where a continuous film is formed. This thickness decreases from 210 to 140 $\AA$ when increasing the pulsing frequency for a constant amount of material deposited per pulse or when increasing the amount of material deposited per pulse and the energy of the film forming species for a constant

\footnotetext{
${ }^{3}$ Corresponding author
} 
Time-domain and energetic bombardment effects on nucleation and coalescence of thin films

pulsing frequency. Estimations of adatom lifetimes and the coalescence times show that there are conditions at which these times are within the range of the modulation of the vapor flux. Thus, nucleation and coalescence processes can be manipulated by changing the temporal profile of the vapor flux. We suggest that other than for elucidating the atomistic mechanisms that control pulsed growth processes, the interplay between the time scales for diffusion, coalescence and vapor flux pulsing can be used as a tool to determine characteristic surface diffusion and island coalescence parameters.

PACS: 68.55.A- Thin Film Structure and Morphology, 68.35.Fx Diffusion; interface formation, 68.43.Jk Diffusion of adsorbates, kinetics of coarsening and aggregation, 81.15.-z Methods of deposition of thin films and coatings; film growth and epitaxy, 81.15.Cd Deposition by sputtering Submitted to: Journal of Physics D: Applied Physics

\section{Introduction}

Thin film formation occurs in three stages; island nucleation and growth, island coalescence and continuous film growth. The distinction between those stages is particularly pronounced during deposition of metallic films on amorphous substrates, where the weak film-substrate interaction results in a Volmer-Weber growth mode [1]. The island density achieved during the nucleation stage and the efficiency of the island coalescence are decisive for the microstructural evolution during growth and hence for film properties, such as roughness, grain size, film thickness at percolation (i.e. when the film is conducting over macroscopic distances) and thickness at which a continuous film is formed [2]. The island number density, $N$, is largely determined by the kinetic conditions during growth through the relation [3]

$$
N \propto\left(\frac{F}{D}\right)^{\chi}
$$


Time-domain and energetic bombardment effects on nucleation and coalescence of thin films

where $\chi$ is a scaling exponent in the range 0.33 to $0.6, F$ is the deposition (vapor) flux and $D$ is the diffusivity of surface atoms (adatoms). $D$ depends exponentially on the growth temperature $T$ and the energy barrier for adatom surface diffusion, $E_{D}$, through the expression [3]

$$
D=\frac{1}{4} \ell^{2} v_{0} \exp \left(-\frac{E_{D}}{k_{B} T}\right)(2)
$$

where $\ell$ is the distance between two neighboring diffusion sites, $k_{B}$ the Boltzmann constant and $v_{0}$ is the so-called attempt frequency. From Eq. (2) it is evident that relatively small variations of $T$ and $E_{D}$ induce relatively large changes in $N$. This is in contrast to $F$, which needs to be varied over many orders of magnitude to affect $N$ to the same degree. Another way to tune the nucleation density is by modulating a constant time-averaged deposition flux in pulses of welldefined width and period [4]. The effect of modulation on nucleation is determined by the relation between the adatom lifetime - i.e. the mean time that an adatom needs to get incorporated into a stable island — the pulse width and the pulse period [4]. Moreover, a modulated flux of atoms implies that the arrival of these atoms occurs over a short period of time. The latter, depending on the temporal profile of the modulation, may lead to a several orders of magnitude increase in the instantaneous adatom arrival rate at the substrate [5,6] and, according to Eq. (1), affect the island density. Island coalescence can also be affected by pulsed deposition fluxes depending on the relation between characteristic time for coalescence and time scale of the modulation of the vapor flux [7].

It is therefore evident that knowledge of the characteristic adatom diffusion and coalescence times is an essential component in utilizing pulsed vapor fluxes for tailor-made film growth. One way to determine these quantities is by synthesizing films using a deposition flux with a wellcontrolled temporal profile and subsequently looking for its fingerprints on the film morphological evolution. Deposition fluxes can be modulated by periodic shuttering of a continuously operating vapor source or by periodic vapor generation, realized for instance by 
Time-domain and energetic bombardment effects on nucleation and coalescence of thin films

pulsed laser deposition (PLD) or pulsed sputtering. PLD processes have been already used to grow Ag films on $\mathrm{SiO}_{2}$ and mica substrates and highlight the time-domain effect of the deposition flux on film coalescence [7]. Moreover, the PLD process generates a highly ionized deposition flux with ion energies up to $300 \mathrm{eV}$ [8]. These energetic ions affect both surface and subsurface layers providing added means for controlling film nucleation and growth [9]. However, the broad IEDFs make identification and understanding of the atomistic mechanisms that along with the modulated vapor flux determine the film microstructural evolution a non-trivial task. In the present study, we separate and establish experimentally the effect of the time-domain and energetic bombardment on the nucleation and coalescence of thin metal films on amorphous substrates. This is achieved by generating pulsed ionized vapor fluxes with much narrower (as compared to PLD) energy distribution functions (up to several tens of $\mathrm{eV}$ ) using a high power impulse magnetron sputtering (HiPIMS) discharge [10-16]. In situ plasma diagnostics and particle transport simulations show that the time-domain of the deposition flux and its energy can be controlled independently. Combining this with in situ monitoring of the deposition of Ag films on $\mathrm{SiO}_{2}-\mathrm{Ag} / \mathrm{SiO}_{2}$ is a well-established archetype system for studying Volmer-Weber growth — and estimations of the adatom diffusion and coalescence times, it is shown that the morphological evolution upon film growth is determined by the effect of the modulated flux on both nucleation and coalescence.

\section{Experimental strategy and procedures}

\subsection{Film synthesis}

Films were deposited from a Ag target (purity 99.99\%) with a diameter of $75 \mathrm{~mm}$ and a thickness of $6 \mathrm{~mm}$ on electrically floating $100 \pm 20 \mu \mathrm{m}$ thick $\mathrm{Si}(100)$ wafers covered with a native $\mathrm{SiO}_{2}$ layer ( 20 Å thick), as well as on electrically floating $500 \mu$ m thick Si (100) wafers covered by a

$3000 \AA$ thick thermally grown $\mathrm{SiO}_{2}$ layer. No intentional substrate heating was used during deposition, while the cathode-to-substrate distance was $12.5 \mathrm{~cm}$ in order to minimize the radiative 
Time-domain and energetic bombardment effects on nucleation and coalescence of thin films

heating of the substrates from the Ag target. Moreover, the cathode formed an angle of 40 degrees with respect to the substrate normal. Depositions were performed in a vacuum chamber with a base pressure below $1.3 \times 10^{-6} \mathrm{~Pa}$. The pressure of the buffer gas (Ar) during film deposition was $0.66 \mathrm{~Pa}$.

In the HiPIMS process the energy of the ionized species, their fraction in the sputtered vapor (degree of ionization) and the amount of material sputtered during a single pulse (which in turn determines the instantaneous arrival rate of film forming species at the substrate) are largely determined by the pulse amplitude, i.e., the energy delivered during the pulse $\left(E_{p}\right)[13,16]$. Therefore, films were deposited using pulses of a constant width of $50 \mu$ s at various values of $E_{p}$ and pulsing frequencies $f$. The latter quantity was altered seeking to change the temporal profile of the vapor flux to the substrate. Two sets of samples were grown; in the first set $E_{p}$ was kept constant at $20 \pm 2 \mathrm{~mJ}$ and $f$ was varied from 50 to $1000 \mathrm{~Hz}$, while in the second set $f$ was fixed at $50 \mathrm{~Hz}$ and $E_{p}$ was varied from $20 \pm 2 \mathrm{~mJ}$ to $1.20 \pm 0.01 \mathrm{~J}$. This growth strategy was implemented aiming at separating the effect of modulation of the vapor generation on the nucleation of $\mathrm{Ag}$ from the effect of the other factors, i.e. energetic bombardment and instantaneous deposition rate. All HiPIMS depositions were performed using an $\mathrm{HiP}^{3}$ power generator (Solvix SA). The time dependent target current and voltage waveforms during the HiPIMS process were measured by a home-made current transducer and a voltage divider, respectively, and were used to calculate $E_{p}$. For the constant $E_{p}$ deposition set, the time-dependent square voltage pulses were found to vary between 456 and $408 \mathrm{~V}$ (for the frequency range 50 to $1000 \mathrm{~Hz}$ ) resulting in current waveforms with peak values between 2.50 and 1.58 A. In the case of the constant $f$ deposition set, voltage varied between 456 and $800 \mathrm{~V}$ with peak currents being between 2.50 and $41.2 \mathrm{~A}$ in the $E_{p}$ range from $20 \mathrm{~mJ}$ to $1.20 \mathrm{~J}$. All target current waveforms exhibited sharp current rise after plasma ignition then settling at nearly constant value lasting until the end of the pulse. Variation of $E_{p}$ and $f$ affects the time-averaged deposition rate, i.e. the time-averaged deposition flux $F$. To 
Time-domain and energetic bombardment effects on nucleation and coalescence of thin films

investigate any effect of $F$ on the nucleation a series of Ag films were grown using the continuous deposition process direct current magnetron sputtering (DCMS) for time-averaged deposition rates within the range of the values achieved for the HiPIMS grown films. The DCMS depositions were performed using an Advanced Energy MDX 1K power generator (Advanced Energy Industries Inc.) operated at a constant current mode with current values between 20 and $70 \mathrm{~mA}$ corresponding to target voltages between 270 and $284 \mathrm{~V}$. Deposition rate values were calculated from the film thicknesses, which were obtained by X-ray reflectometry (XRR) measurements performed in a Philips Bragg-Brentano diffractometer.

\subsection{Plasma and deposition flux characterization}

The effect of the deposition parameters on the plasma chemistry, energetics and temporal profile was investigated by means of time- and energy-resolved mass spectrometry using a Hiden PSM003 spectrometer (Hiden Analytical Ltd.) with an electrically floating orifice. The target-toorifice distance was $19 \mathrm{~cm}$. The electrostatic lenses in the spectrometer were tuned to maximize the transfer of ${ }^{36} \mathrm{Ar}^{+}$and ${ }^{107} \mathrm{Ag}^{+}$as the transfer function of the spectrometer varies over large mass intervals. The acceptance angle of the spectrometer is energy dependent and decreases with the energy of the ions [17]. This implies that mass spectrometry measurements tend to overestimate the flux of low energy ions compared to the flux of high energy ions. Thus, the ion energy distribution functions (IEDFs) recorded in the present study are merely used to probe the effect of the deposition conditions on the plasma chemistry, energetics and temporal profile on a qualitative level. Time resolved IEDFs were measured for ${ }^{36} \mathrm{Ar}^{+}$and ${ }^{107} \mathrm{Ag}^{+}$ions. The IEDFs were recorded in $200 \mu$ s steps starting $50 \mu$ s after pulse initiation over a total period of $21.2 \mathrm{~ms}$ to cover the flux evolution over one full pulse cycle for all pulsing frequencies. The energy step of the measurements was $0.5 \mathrm{eV}$. To improve the measurement statistics, each energy point was integrated over 15 pulses for the frequency series and over 5 pulses for the pulse power series. 
Time-domain and energetic bombardment effects on nucleation and coalescence of thin films

The IEDFs were integrated over the energy intervals for each time step in order to quantify the time evolution of the ion fluxes.

Monte-Carlo simulations were employed to study the vapor generation and transport from the target to the substrate. The interactions of the plasma with the Ag target were simulated using the TRIM code [18]. Ar ions with an energy corresponding to the cathode voltage for each simulated condition were used as bombarding species and the initial energy and angular distribution of the sputtered Ag atoms were obtained. These distributions were used as input together with the experimental gas pressure and chamber geometry to simulate the interactions of the sputtered species with the buffer gas (Ar) en route to the substrate (employing SIMTRA) [19] and obtain the energy and the time-of-flight of the sputtered species arriving at the substrate.

\subsection{Study of film nucleation and coalescence}

The nucleation and coalescence characteristics of the Ag films grown at the various process conditions were studied by determining the thickness at which the coalescence is completed $\left(d_{\text {coal }}\right)$, i.e. the film thickness at which the continuous film growth begins. This was achieved by in situ monitoring of the intrinsic (growth) stress evolution and the optical properties of the films.

Intrinsic stresses were measured for films deposited on the $100 \pm 20 \mu \mathrm{m}$ thick Si (100) substrates covered with the native $\mathrm{SiO}_{2}$ layer employing a multi-beam optical stress sensor (k-Space Associates Inc.) with an angle of incidence of approximately 60 degrees from the substrate surface normal. The average stress-thickness product of the film was calculated by measuring changes of the substrate curvature during film deposition and implementing the modified Stoney equation [20,21]. The sign of the slope of the stress-thickness product curve as a function of the film thickness gives the sign of the stress where, by convention, negative values signify compressive stress and positive values signify tensile stress. For films grown under high mobility 
Time-domain and energetic bombardment effects on nucleation and coalescence of thin films

conditions (encountered when depositing Ag at room temperature) a compressive-tensilecompressive stress evolution is commonly observed upon film thickening [22-26]. It has been shown $[25,26]$ that the tensile-to-compressive transition coincides with the completion of the coalescence and the formation of a continuous film. This transition can be seen in Figure 1, where the stress evolution in a film deposited at $f=50 \mathrm{~Hz}$ and $E_{p}=20 \mathrm{~mJ}$ is plotted as a function of the nominal film thickness. The latter is calculated by considering that the film grows with a constant rate, as determined from films with thicknesses on the order of $250 \AA$ (considered to be within the continuous film growth regime), throughout all stages. The coalescence completion thickness is then determined to be the nominal thickness that corresponds to the maximum in the stress evolution (indicated by the vertical dashed line in Figure 1).

The evolution of the optical properties was monitored in situ during deposition by means of spectroscopic ellipsometry. Ellipsometric angles ( $\Psi$ and $\Delta$ ) were measured during deposition of films on the $500 \mu$ m thick $\mathrm{Si}(100)$ substrates covered by a $3000 \AA$ thick thermally grown $\mathrm{SiO}_{2}$ layer in the spectral range 1.6 to $3 \mathrm{eV}$ using a Woolam M88 rotating analyzer ellipsometer (J.A. Woolam Co. Inc.) at an angle of incidence of 65 degrees measured from the substrate normal. The data were analyzed using a three-phase model consisting of substrate, film and vacuum. The optical properties of the substrate were determined by recording a spectrum prior to the initiation of the deposition. The dielectric function of the film was parameterized using a dispersion relation consisting of a Drude term that describes optical response of ideal metals [27] as given by the expression

$$
\tilde{\varepsilon}(\omega)=\varepsilon_{\infty}-\frac{\omega_{p}^{2}}{\omega^{2}-i \Gamma_{D} \omega}(3)
$$

In Eq. (3) $\omega$ is the photon energy of the incoming light, $\omega_{p}$ is the plasma frequency of the free electrons, $\Gamma_{D}$ is the electron damping factor (a measure of the free electron relaxation time) and $\varepsilon$ 
Time-domain and energetic bombardment effects on nucleation and coalescence of thin films

$\infty$ is a background constant larger than one that accounts for contribution of high energy interband transitions to the dielectric function that are not included in the model. The parameters $\omega_{p}, \Gamma_{D}$ and $\varepsilon_{\infty}$, were varied along with the film thickness to fit the model to the experimental data and the film resistivity, $\rho$, was calculated using the formula [28]

$$
\rho=\left(\frac{1}{\varepsilon_{0}}\right) \frac{\Gamma_{D}}{\omega_{p}^{2}}(4)
$$

where $\varepsilon_{0}$ is the permittivity of the free space. Then $\rho$ was plotted, at each time of the deposition, as a function of the nominal film thickness calculated using the same procedure as for the in-situ stress measurements. A typical curve (for a sample grown at $f=50 \mathrm{~Hz}$ and $E_{p}=20 \mathrm{~mJ}$ ) is shown in Figure 2 in which it is seen that below a critical nominal thickness value (indicated by the vertical dashed line in Figure 2) $\rho$ deviates from a steady state behaviour. The critical thickness is taken to correspond to $d_{\text {coal }}$, i.e. the transition from the coalescence to the continuous growth stage [29]. It is worth mentioning that the steady-state $\rho$ value in Figure 2 corresponds to $\omega_{p} \sim 9.2 \mathrm{eV}$ and $\Gamma_{D} \sim 0.02 \mathrm{eV}$ which is very close to the values for bulk Ag [30].

\section{Results}

\subsection{Temporal profile, energy and composition of ionized and neutral species}

Time-resolved ion energy distribution functions (IEDFs) of $\mathrm{Ag}^{+}$ions recorded from a discharge operated at $E_{p}=20 \mathrm{~mJ}$ and $f=100 \mathrm{~Hz}$ are plotted in Figure. 3. The presented data correspond to one pulsing period, i.e. the first IEDF was recorded $50 \mu$ s from the pulse initiation and the last IEDF $50 \mu$ s after the onset of the next pulse. Furthermore, only every fifth recorded IEDF is presented for clarity. The $\mathrm{Ag}^{+}$ions $50 \mu$ s after the initiation of each pulse exhibit IEDFs that range from 1 to $20 \mathrm{eV}$. In all other time steps significantly narrower IEDFs with energies of approximately $2 \mathrm{eV}$ are measured. Similar behaviour with respect to the temporal evolution of the IEDFs was observed at pulsing frequencies other than $100 \mathrm{~Hz}$. 
Time-domain and energetic bombardment effects on nucleation and coalescence of thin films

The time evolution of the integrated $\mathrm{Ag}^{+}$ion flux at $E_{p}=20 \mathrm{~mJ}$ for $f=100,400$ and $1000 \mathrm{~Hz}$ is plotted in Figure 4. The ion flux exhibits periodic maxima coinciding with the power pulses, with a delay due to the time-of-flight from the cathode to the spectrometer. A periodic oscillation of the $\mathrm{Ag}^{+}$flux, consistent with the power pulsing frequency, was also found by time-resolved IEDFs recorded from discharges operating at $f=50 \mathrm{~Hz}$ and $E_{p}$ values varying from $20 \mathrm{~mJ}$ to $1.20 \mathrm{~J}$ (not shown). It should also be pointed out here that measurements showed that at all conditions the intensity of $\mathrm{Ag}^{2+}$ ions was less than $5 \%$ of the $\mathrm{Ag}^{+}$ion intensity. Therefore doubly ionized species are not considered in the subsequent discussion.

TRIM simulations showed that the vast majority of the sputtered Ag species exhibits energies of several tens of $\mathrm{eV}$, while only a small percent reaches energies up to several hundreds of $\mathrm{eV}$. This energy distribution results in a maximum average energy (among the various deposition conditions used as input for the simulations) of $21.2 \mathrm{eV}$, that decreases down to $2.3 \mathrm{eV}$ after the sputtered Ag atoms interact with the buffer gas atoms during transport to the substrate (as simulated using the SIMTRA code). The statistical distribution of the time-of-flight for the Ag species from the target to the substrate was not found to vary significantly for the different deposition conditions, revealing that $90 \%$ of the sputtered species arrive at the substrate within 15 ms from their generation. Assuming that the sputtered vapor is generated instantaneously, one thousand time-of-flight distribution functions were superimposed and added for various pulsing frequencies (Figure 5 for 100, 400 and $1000 \mathrm{~Hz}$ ) to estimate the steady-state temporal profile of neutral deposition flux to the substrate. Similarly to the ion flux (Figure 4), the flux of neutral species exhibits periodic maxima consistent with the power pulsing frequencies.

Figure 6 compares $\mathrm{Ag}^{+}$IEDFs recorded $50 \mu$ s after the pulse initiation from a discharge of $E_{p}=20$ $\mathrm{mJ}$ and $f=100$ and $1000 \mathrm{~Hz}$ (solid and dashed line, respectively). It is seen that both frequencies yield identical IEDFs with respect to the shape, while small differences in the measured 
Time-domain and energetic bombardment effects on nucleation and coalescence of thin films

intensities are observed. The effect of $E_{p}$ for a constant frequency $(f=50 \mathrm{~Hz})$ on $\mathrm{Ag}^{+}$IEDFs is shown in Figure 7. It is seen that an $E_{p}$ value of $1.20 \mathrm{~J}$ (dashed-dotted line) results in broader IEDF as compared to that obtained at $20 \mathrm{~mJ}$ (dashed line) signifying an increase of the $\mathrm{Ag}^{+}$ion energy. Regarding $\mathrm{Ar}^{+}$ions, measurements revealed that at all process conditions relatively narrow IEDFs centered on $\sim 1 \mathrm{eV}$ are obtained. Moreover, the mass spectrometry data revealed that the $\mathrm{Ar}^{+} / \mathrm{Ag}^{+}$integrated intensity ratio was in the order of 4 at all deposition conditions. This enables us to draw the qualitative conclusion that the flux of $\mathrm{Ar}^{+}$ions to the growing film is larger than that of $\mathrm{Ag}^{+}$ions.

XRR measurements showed that the time averaged deposition rate increased from 0.05 to 1.05 $\AA / \mathrm{s}$ when the frequency was increased from 50 to $1000 \mathrm{~Hz}$ (for $E_{p}=20 \mathrm{~mJ}$ ). It should be also pointed out that despite the increase of the time-averaged deposition rate a nearly constant deposition rate of $0.001 \AA$ per pulsing period was obtained for this set of depositions. Increasing $E_{p}$ from $20 \mathrm{~mJ}$ to $1.20 \mathrm{~J}$ (at $f=50 \mathrm{~Hz}$ ) leads to a deposition rate increase to $1.45 \AA /$ s corresponding to $0.029 \AA$ per pulsing period.

\subsection{Nucleation and coalescence characteristics}

The effect of the discharge pulse frequency on $d_{\text {coal }}$ - determined by in situ monitoring of the intrinsic stress evolution - for $E_{p}=20 \mathrm{~mJ}$ shown in Figure 8 (a). $d_{\text {coal }}$ decreases from $\sim 210 \AA$ to 140 A upon increasing pulsing frequency up to $500 \mathrm{~Hz}$ above which no significant change in $d_{\text {coal }}$ is observed. Figure 8 (b) presents the change of $d_{\text {coal }}$ as function of deposition rate (obtained in the same frequency range as the one used in Figure. 8(a)) for films deposited at $E_{p}=20 \mathrm{~mJ}$. The results for films grown by DCMS within the same deposition rate range are also shown for reference. It can be seen that variation of the deposition rate from 0.2 to $1 \AA$ /s when a continuous deposition flux is employed does not affect $d_{\text {coal }}$, as opposed to the case of a pulsed flux. The effect of $E_{p}$ on $d_{\text {coal }}$ for a constant frequency of $50 \mathrm{~Hz}$ is shown in Figure. 8 (c). An increase of $E_{p}$ 
Time-domain and energetic bombardment effects on nucleation and coalescence of thin films

from 20 to $60 \mathrm{~mJ}$ results in a decrease of $d_{\text {coal }}$ from 210 to $160 \AA \AA$. Further increase of $E_{p}$ does not significantly affect $d_{\text {coal }}$.

Figure 9 presents the effect of pulsing frequency (for $E_{p}=20$ mJ, Figure. 9 (a)) and $E_{p}($ for $f=50$ Hz, Figure. 9 (b)) on $d_{\text {coal }}$, as determined by spectroscopic ellipsometry. The corresponding values from the intrinsic stress measurements are also plotted for comparison. It is seen that the trends obtained by the stress analysis are confirmed by ellipsometry; $d_{\text {coal }}$ decreases from $\sim 220 \AA$ to $\sim 150 \AA$ when increasing $f$ or $E_{p}$ to $500 \mathrm{~Hz}$ and $200 \mathrm{~mJ}$ respectively, above which no significant change can be seen.

\section{Discussion}

The results presented in Figures 3 and 4 show that the flux of the ionized sputtered species is modulated. The same is the case for the Ag neutrals as suggested by the simulation results in Figure 5. A large fraction of the film forming species (both neutral and ionized) arrives at the substrate in pulses with frequencies that are consistent with the frequency at which the power is applied to the target. The effect of a modulated deposition flux on the nucleation density depends on the relation between the time domain of the modulation (pulse width and period) and the adatom lifetime, $\tau_{D}$. The latter can be calculated by the expression [3]

$$
\tau_{D} \approx \frac{1}{N D}(5)
$$

where $D$ is the surface diffusion coefficient, given by Eq. (2), and $N$ the total number density of islands on the surface. To estimate $\tau_{D}$ we assume that the $\mathrm{SiO}_{2}$ surface is covered by up to 0.1 monolayers (ML) of Ag. This coverage, $\Theta$, is a typical value at which a steady state island density is achieved [3]. The nuclei density in the steady-state nucleation regime can be calculated by the equation [3]

$$
N=\frac{n}{\Omega^{1 / 3}}\left(\frac{F}{D}\right)^{1 / 3}
$$


Time-domain and energetic bombardment effects on nucleation and coalescence of thin films

In Eq. (6) $n$ is a dimensionless pre-factor that is a function of the coverage and the critical nucleus size which in the case of irreversible nucleation (i.e., a dimer is the smallest stable atomic configuration on the surface of the growing film) and low coverage has been estimated to obtain a maximum value of 0.25 [31]. The parameter $\Omega$ denotes the area of an adsorption site and can be estimated as $\Omega=\ell^{2}$, where $\ell$ is the distance between nearest atomic neighbors, here for simplicity taken to be $2 \AA$. To calculate $D$, Eq. (2), an attempt frequency, $v_{0}$, of $5 \times 10^{12} \mathrm{~s}^{-1}$ is used [3], while the energy barrier for surface diffusion $E_{D}$, according to the literature, is in the range 0.3 to 0.45 $\mathrm{eV}$ [32]. The most common termination for fcc metals (like Ag) is the (111) surface and the Ag (111) interplanar distance is $2.36 \AA$. Thus, a vapor flux in the range 0.05 to $1.45 \AA /$ s (section 3.1 ) corresponds to 0.02 to $0.62 \mathrm{ML} / \mathrm{s}$. By using an average value of $0.31 \mathrm{ML} / \mathrm{s}$ along with $T=300 \mathrm{~K}$, as well as the $E_{D}, n$ and $\Omega$ values mentioned above an island density, $N$, of the order of $10^{13} \mathrm{~cm}^{-2}$ can be calculated from Eq. (6). 1 ML of (111) Ag contains $\sim 10^{15}$ atoms $/ \mathrm{cm}^{2}$ which means that 0.1 ML corresponds to an atom density of $\sim 10^{14}$ atoms $/ \mathrm{cm}^{2}$ which for an island density of $10^{13} \mathrm{~cm}^{-2}$ means that the maximum average islands size is $\sim 10$ atoms. Thus, $\tau_{D}$ can be calculated from Eq. (5) assuming for simplicity that $N=\Theta / 10$ at all $\Theta$ values. Figure 10 presents $\tau_{D}$ as a function of $\Theta$ in the form of a shaded area limited by solid line boundaries determined by the $E_{D}$ ( 0.3 to 0.45 $\mathrm{eV}$ ) and $\Theta$ values (up to 0.1 ) used for the calculation. The results indicate that there are deposition conditions at which $\tau_{D}$ lies within the time scale of the modulation of the deposition flux as determined by the range of the pulsing period used in the experiments (from 1 to $20 \mathrm{~ms}$ as indicated by the dashed horizontal lines in Figure 10). Thus, one can identify pulsing frequencies for which the adatoms from the previous pulse would disappear from the surface of the growing film before the next pulse arrives, resulting in an island density determined by the instantaneous flux in the pulse. A decrease of the pulsing period (i.e., an increase of the pulsing frequency) to values comparable to the adatom lifetime would result in an increase of the adatom density, since the adatoms are no longer depleted between the pulses. This, in turn, leads to a shorter adatom 
Time-domain and energetic bombardment effects on nucleation and coalescence of thin films

diffusion length and to an increase of the island density. Thus, a continuous film would be formed at smaller nominal thicknesses, which can explain the lower coalescence completion thickness for increasing pulsing frequency (Figures 8 (a) and 9 (a)).

Another phenomenon that can influence the value of $d_{\text {coal }}$ is island (nuclei) coalescence. For spherical islands of comparable size coalescence is a surface diffusion driven process with a characteristic time given by the relation $[33,34]$

$$
\tau_{c o d} \approx \frac{k_{B} T R^{4}}{D_{s} V_{a}^{4 / 3}}(7)
$$

In Eq. (7) $R$ is the size of the coalescing islands, $D_{s}$ is the diffusivity of the atoms on the island surface, $\gamma$ is the surface free energy of the island and $V_{a}$ is the atomic volume. We calculated $\tau_{\text {coal }}$ for $R$ in the range 5 to $150 \AA$ using a $\gamma$ value of $0.071 \mathrm{eV} / \AA^{2}$ for the $\mathrm{Ag}$ (111) surface [35] and $V_{a}=a^{3}$, where $a=2.8 \AA$ is the nearest neighbor distance on the $\mathrm{Ag}$ (111) surface [7]. $D_{s}$ was calculated from Eq. (2) using a surface diffusion barrier, $E_{D}$, of $0.26 \mathrm{eV}$. This value is the average of the step-edge barriers for A and B steps on Ag (111), 0.22 and $0.3 \mathrm{eV}$ respectively [36,37], and is used since faceting occurs at relatively low temperatures making step diffusion the rate limiting process [7]. The results of the calculations are presented in Figure 10, where it can be seen that above a certain island radius the coalescence time falls within the time domain of the modulated deposition flux (indicated by the dashed horizontal lines in Figure 10). This implies that by increasing the frequency (i.e., decreasing the pulsing period) new adatoms are supplied to the surface of the growing film prior the time required for the completion of coalescence events. These adatoms can be either incorporated to the coalescing cluster increasing $\tau_{\text {coal }}$ or cause growth of islands which might impinge on a coalescing cluster. This retards the coalescence of isolated clusters causing the formation of elongated clusters that is the first step towards the formation of a continuous film [7]. Hence, the decrease of $d_{\text {coal }}$ upon increasing pulsing frequency, 
Time-domain and energetic bombardment effects on nucleation and coalescence of thin films

Figures 8 (a) and 9 (a), can be attributed both to an increase of nucleation density at the initial film formation stages as well as to retardation or interruption of cluster coalescence at subsequent growth stages.

It can also be seen from the results presented in Section 3.1 that changes of the flux modulation (facilitated by changes in the pulsing frequency at a constant pulse power) do not influence the energy of the ionized sputtered species, as shown in Figure 6. In addition, a mean $\mathrm{Ag}^{+}$ion energy of $\sim 4 \mathrm{eV}$ with $\sim 5 \%$ of the ions having energies larger than $10 \mathrm{eV}$ can be calculated for all pulsing frequencies with $E_{p}=20 \mathrm{~mJ}$. As mentioned in Section 2, the substrates were electrically floating during deposition. Thus, ionized species are accelerated to the substrate by the floating potential which has values of approximately $-10 \mathrm{~V}$ [38,39], i.e. ionized species $\left(\mathrm{Ar}^{+}\right.$and $\left.\mathrm{Ag}^{+}\right)$impinging on the substrate have energies above $10 \mathrm{eV}$, which are significantly larger than those of sputtered atoms $(\sim 2 \mathrm{eV})$. The ratio of the integrated ion $\left(\mathrm{Ag}^{+}, \mathrm{Ar}^{+}\right)$flux (calculated from the temporal evolution of the IEDFs, see e.g. Figure. 4) to the deposition rate (reported in Section 3.1) decreases by nearly a factor of 3 when increasing the pulsing frequency from 50 to $1000 \mathrm{~Hz}$. This signifies a decrease of the number of energetic species available to provide additional energy to the surface during deposition. Bombardment of the film surface with $\mathrm{Ag}^{+}$and $\mathrm{Ar}^{+}$ions in the energy range in the present study mainly causes enhancement of adatom surface diffusion [9]. However, the total number of ions in the deposition flux for $E_{p}$ of the order of $20 \mathrm{~mJ}$ is expected to be rather low (typically less than $10 \%$ of the total number of species) [40]. Bombardment by ions in this energy range is mainly expected to affect the topmost atomic layer enhancing adatom diffusion, effectively leading to a larger diffusivity [41,42]. This should result in a lower island density and lower coalescence times both resulting in a larger film thickness at the completion of coalescence. Thus, we suggest that the decrease of $d_{\text {coal }}$ upon increasing frequency, Figures 8 (a) and 9 (a), cannot be attributed to changes in the conditions of energetic bombardment during growth. 
Time-domain and energetic bombardment effects on nucleation and coalescence of thin films

According to Eq. (1), another factor that can affect nucleation characteristics in a pulsed deposition process is the instantaneous arrival rate of the vapor. Taking into account the average rate values $(F)$ for the frequency range 50 to $1000 \mathrm{~Hz}$ and assuming, for simplicity, that most of the deposition takes place during the intense material pulse $\left(t_{\text {pulse }} \sim 200 \mu\right.$ s according to Figures 4 and 5) an upper limit for the instantaneous deposition rate $\left(F^{\text {inst }}\right)$ of $\sim 50 \AA / \mathrm{s}$, constant for all frequencies, can be calculated from the following expression

$$
F^{i n s t}=\frac{F}{f \cdot t_{p u s e}}(8)
$$

The constant value of $F^{\text {inst }}$ means that the reason for the decrease in $d_{\text {coal }}$ upon increasing frequency observed in Figure 8 (a) and Figure 9 (a) does not lie on the amplitude of the deposition flux but merely on its temporal profile.

Increasing $E_{p}$ from $20 \mathrm{~mJ}$ to $1.20 \mathrm{~J}$ at a constant pulsing frequency results in an increase of the mean energy of the $\mathrm{Ag}^{+}$ions from $\sim 4 \mathrm{eV}$ with a relatively narrow IEDF to $\sim 20 \mathrm{eV}$ with a relatively long high energy tail (as seen in Figure 7). In addition, the total ion flux to deposition rate ratio (as calculated from the temporal evolution of the integrated ion intensity) decreases by a factor of 10. Bombardment by ions in the energy range of several tens of eV may cause creation of surface defects as well as atomic cluster dissociation $[42,43]$ that can lead to an increase of the nuclei density. At the same time, $F^{i \text { int }}$ increases from $\sim 50 \AA / \mathrm{s}$ to $\sim 1500 \AA / \mathrm{s}$ in the $E_{p}$ range $20 \mathrm{~mJ}$ to $1.20 \mathrm{~J}$ which also lead to an increase of the nuclei density according to Eq. (1). Moreover, the results in Figure 8 (b) show that changes $d_{\text {coal }}$ cannot be attributed to changes in the average deposition rate when the latter lies in the range 0.2 to $1 \AA$ /s. Thus, both ion-surface interactions and instantaneous deposition rate can explain the decrease in $d_{\text {coal }}$ observed in Figures 8 (c) and 9 (b) when $E_{p}$ is increased. 
Time-domain and energetic bombardment effects on nucleation and coalescence of thin films

An additional factor capable of affecting film nucleation and coalescence is variation of the substrate temperature due to the interaction of the growing film with the plasma species. Increase of $f$ at a constant $E_{p}$ value or increase of $E_{p}$ at a constant $f$ value results in a larger total flux (ions and neutrals) arriving at the substrate per time unit which may lead to an increase of the actual substrate temperature during deposition. Should the latter happen, adatom mobility would be enhanced yielding lower nuclei density (Eqs. (1) and (2)), while the coalescence time would also be decreased (Eq. (7)). Both lower nuclei density and short coalescence time would lead to a larger coalescence completion thickness. Since the opposite behavior is observed in figures 8 and 9, we can conclude that possible variation of the substrate temperature is not the main factor affecting the microstructural evolution during film growth.

\section{Summary and conclusions}

Experimental routes that allow for separation of effects of vapor flux modulation on the nucleation and growth of $\mathrm{Ag}$ on $\mathrm{SiO}_{2}$ substrates from the effects of bombardment by energetic species and instantaneous deposition rate during the vapor pulses have been established. It has been shown that an increase of the pulsing frequency leads to a decrease of the thickness at which a continuous film is formed. Estimations of the adatom lifetime and island coalescence time have revealed that there are synthesis conditions in which these times lie within the time domain of the modulation deposition flux. Based on this, we argue that an increase of the frequency results in an increase of the adatom and nucleation density and/or retardation of island coalescence thus shifting the formation of a continuous film to lower thicknesses. An increase of the island density was also the explanation for the decrease of the coalescence completion thickness upon increasing the instantaneous vapor flux during the material pulse and the energy of the species impinging on the growing film. The modulated, low energy fluxes generated in the present study along with the results regarding the film morphology can provide data to be used to benchmark theoretical descriptions (e.g. kinetic Monte-Carlo and Molecular Dynamics simulations) of 
Time-domain and energetic bombardment effects on nucleation and coalescence of thin films

growth processes where energetic bombardment and modulation of the vapor flux are key factors. The pulsing frequency dependence on the morphological evolution can also be used as a tool to determine effective diffusion and coalescence times. By measuring and observing the evolution of the island density at different growth stages, conditions at which characteristic times for diffusion and coalescence become comparable to the time-scale of the modulation of the vapor flux can be accurately identified. This, in turn, can be used to determine surface diffusion and/coalescence time constants at various conditions of energetic bombardment.

\section{Acknowledgements}

D.M. and U.H. acknowledge financial support from the Swedish Research Council through contract VR 621-2011-4280. G.A. acknowledges the support from COST Action MP0804 "Highly Ionized Pulsed Plasmas" that has financed the short-term scientific mission (STSM) at Linköping University during which part of this work has been performed. V.E. and K.S. should like to acknowledge the financial support from Linköping University via the " $L i U$ Research Fellows” program. The authors would like to acknowledge Prof. Joe Greene and Prof. Ivan Petrov for helpful discussions.

\section{REFERENCES}

[1] Martin P M (Ed) 2005 Handbook of Deposition Technologies for Films and Coatings Amsterdam Elsevier p. 556

[2] Ohring M 1991 Materials Science of Thin Films San Diego Academic Press p. 383-385

[3] Michely T and Krug J 2005 Island Mounds and Atoms Berlin Springer

[4] Jensen P and Niemeyer B 1997 Surf. Sci. 384 L823

[5] Latteman M, Helmersson U and Greene J E 2010 Thin Solid Films 5185978

[6] Almyras G A, Matenoglou G M, Komnimou Ph, Kosmidis C, Patsalas P and Evangelakis G A 2010 J. Appl. Phys. 107084313 
Time-domain and energetic bombardment effects on nucleation and coalescence of thin films

[7] Warrender J M and Aziz M J 2007 Phys. Rev. B 76045414

[8] Warrender J M and Aziz M J 2007 Phys. Rev B. 75085433

[9] Petrov I, Barna P B, Hultman L and Greene J E 2003 J. Vac. Sci. Technol. A 21 S117

[10] Kouznetsov V, Macak K, Schneider J M, Helmersson U and Petrov I 1999 Surf. Coat. Technol. 122290

[11] Helmersson U, Lattemann M, Böhlmark J, Ehiasarian A P and Gudmundsson J T 2006 Thin Solid Films 5131

[12] Alami J, Bolz S and Sarakinos K 2009 J. All. Comp. 483530

[13] Sarakinos K, Alami J and Konstantinidis S 2010 Surf. Coat. Technol. 2041661

[14] Anders A 2010 Surf. Coat. Technol. 205 S1

[15] Lundin D and Sarakinos K 2012 J. Mater. Res. 27780

[16] Gudmundsson J T, Brenning N, Lundin D and Helmersson U 2012 J. Vac. Sci. Technol. A 30030801

[17] Hamers E A G, van Sark W G J H M, Bezemer J, Goedheer W J and van der Weg W F 1998 Int. J. Mass Spectrom. Ion Processes 17391

[18] Ziegler J F, Biersack J P and Littmark U 1985 The Stopping and Range of Ions in Solids, Pergamon New York http://www.srim.org.

[19] Van Aeken K, Mahieu S and Depla D 2008 J. Phys. D: Appl. Phys. 41205307

[20] Stoney G G 1909 Proc. R. Soc. London, Ser. A 82172

[21] Janssen G C A M, Abdalla M M, van Keulen F, Pujada B R and van Venrooy B 2009 Thin Solid Films 5171858

[22] Abermann R and Koch R 1985 Thin Solid Films 12971

[23] Shull A L and Spaepen F 1996 J. Appl. Phys. 806243

[24] Floro J A, Hearne S J, Hunter J A, Kotula P, Chason E, Seel S C and Thompson C V 2001 J. Appl. Phys. 89, 4886

[25] Abermann R, Koch R and Kramer R 1979 Thin Solid Films 58, 365 
Time-domain and energetic bombardment effects on nucleation and coalescence of thin films

[26] Flöttotto D, Wang Z M, Jeurgens L P H, Bischoff E and Mittemeijer E J 2012 J. Appl. Phys. 112043503

[27] Wooten F 1971 Optical Properties of Solids New York Academic Press p. 52

[28] Ashcroft N W and Mermin N D 1976 Solid State Physics New York Holt, Rinehart and Winston p.8

[29] Patsalas P and Logothetidis S 2003 J. Appl. Phys. 93989

[30] Johnson P B and Christy R W 1972 Phys. Rev. B 64370

[31] Venables J A, Spiller G D T and Hanbücken M 1984 Rep. Prog. Phys. 47399

[32] Kim H C, Alford T L and Allee D R 2002 Appl. Phys. Lett. 814287 and references therein.

[33] Jeffers G, Dubson M A and Duxbury P M 1994 J. Appl. Phys. 755016

[34] Nichols F A and Mullins W W 1965 J. Appl. Phys. 361826

[35] Nada K K, Sahu S N and Behera S N 2002 Phys. Rev. A 66013208

[36] Nelson R C, Einstein T L and Khare S V 1993 Surf. Sci. 295462

[37] Schlösser D C, Morgenstern K, Verheij K, Rosenfeld G, Besenbacher F and Comsa G 2000 Surf. Sci. 46519

[38] Gudmundsson J T, Alami J and Helmersson U 2001 Appl. Phys. Lett. 783427

[39] Stranak V, Drache S, Cada M, Hubicka Z, Tichy M and Hippler R 2011 Contrib. Plasma Phys. 51237

[40] Böhlmark J, Lattemann M, Gudmundsson J T, Ehiasarian A P, Aranda Gonzalvo Y, Brenning N and Helmersson U 2002 Thin Solid Films 5151522

[41] Gilmore C M and Sprague J A 1991 Phys. Rev. B 448950

[42] Adamovic D, Münger E P, Chirita V, Hultman L and Greene J E 2005 Appl. Phys. Lett. 86 211915

[43] Adamovic D, Chirita V, Münger E P, Hultman L and Greene J E 2006 Thin Solid Films 515 2235 
Figures

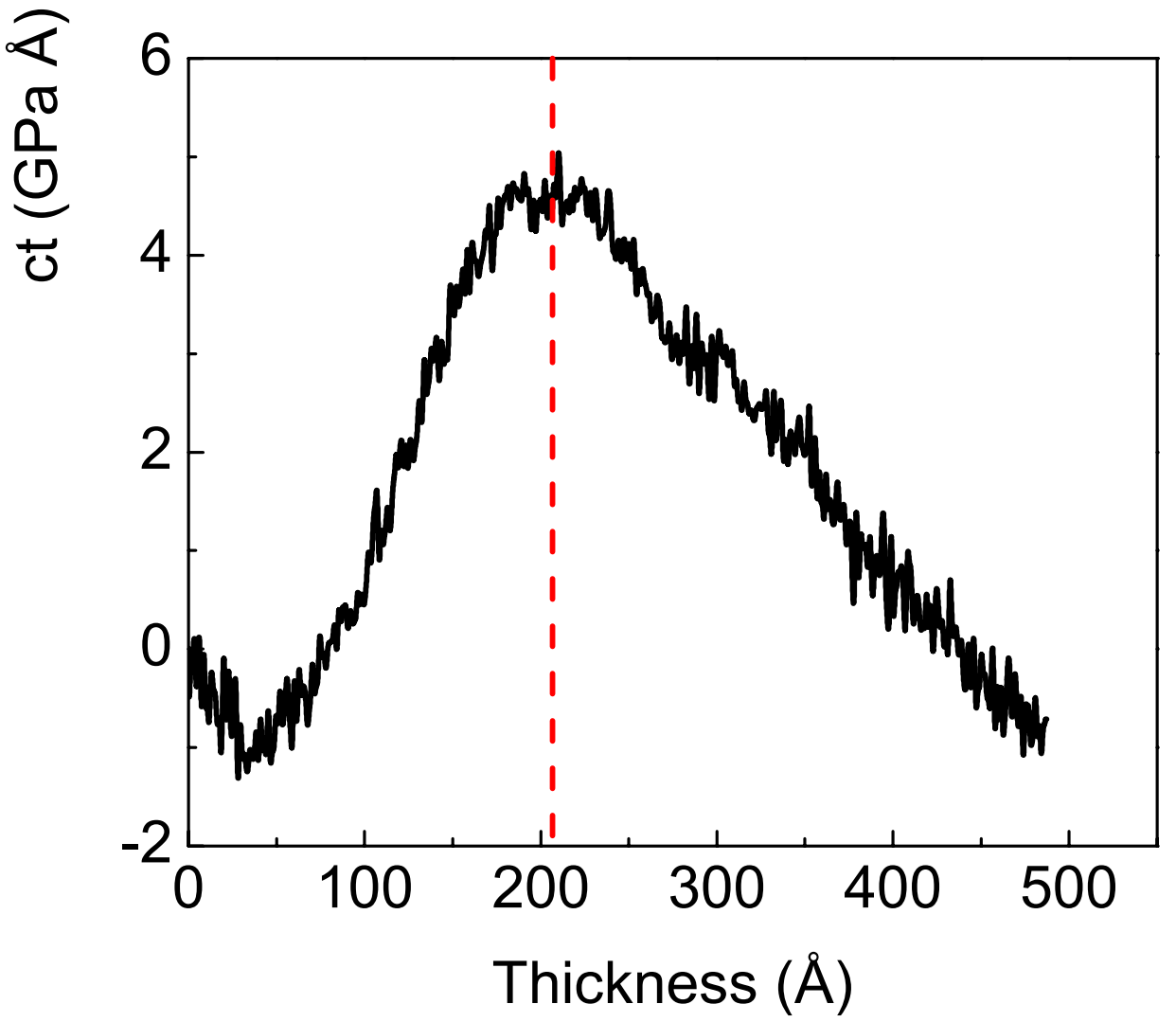

Figure 1. Stress-thickness product as a function of the film nominal thickness for an Ag film deposited at $f=50 \mathrm{~Hz}$ and $E_{p}=20 \mathrm{~mJ}$. The transition from tensile to compressive stress state (indicated by the vertical dashed line) signifies the completion of the coalescence and the onset of the continuous film growth stage. 


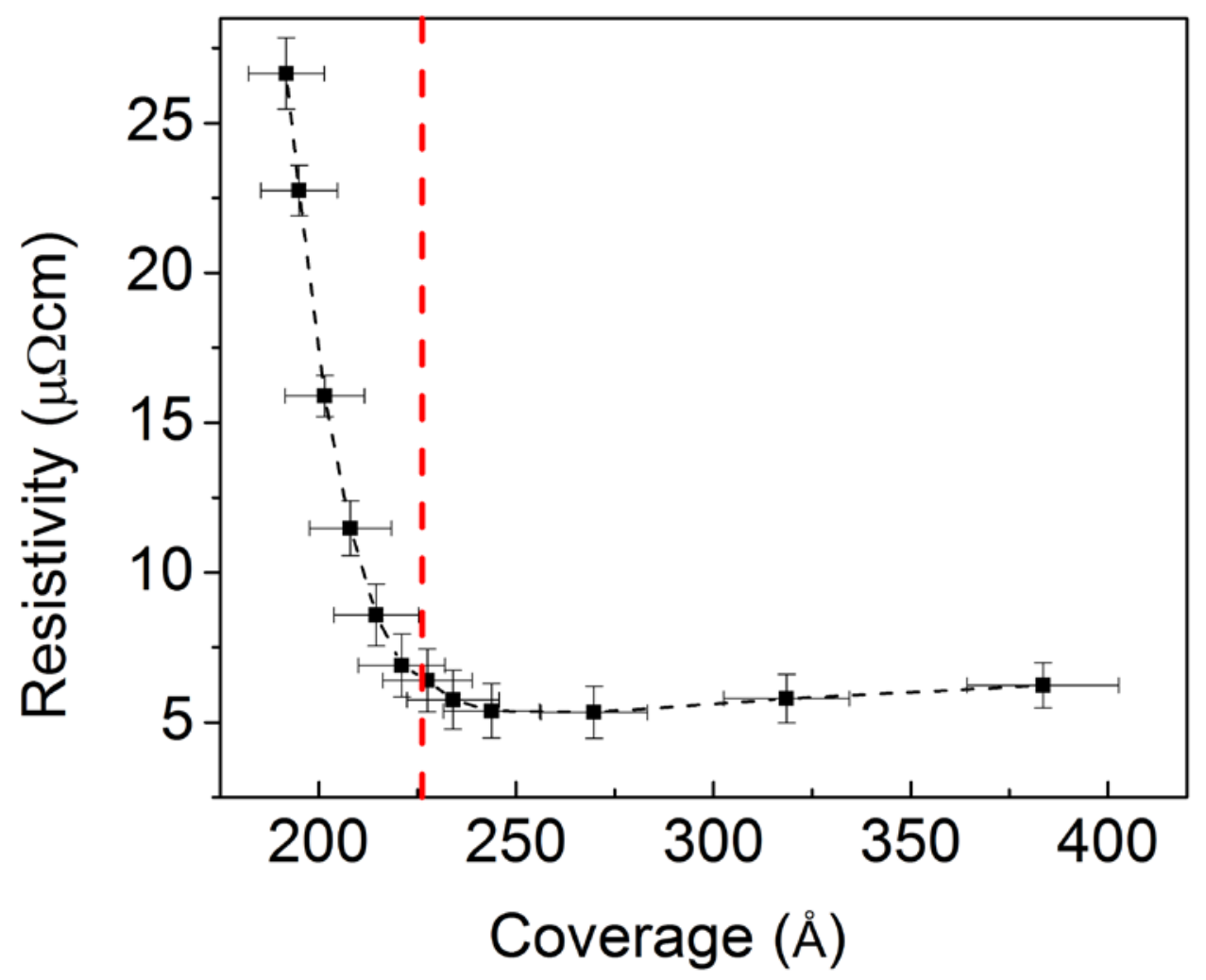

Figure 2. Resistivity, $\rho$, of an Ag film deposited at $f=50 \mathrm{~Hz}$ and $E_{p}=20 \mathrm{~mJ}$ as a function of the film nominal thickness. The deviation of the resistivity from the steady state behaviour (indicated by the vertical dashed line) signifies the transition between coalescence and continuous film growth. 


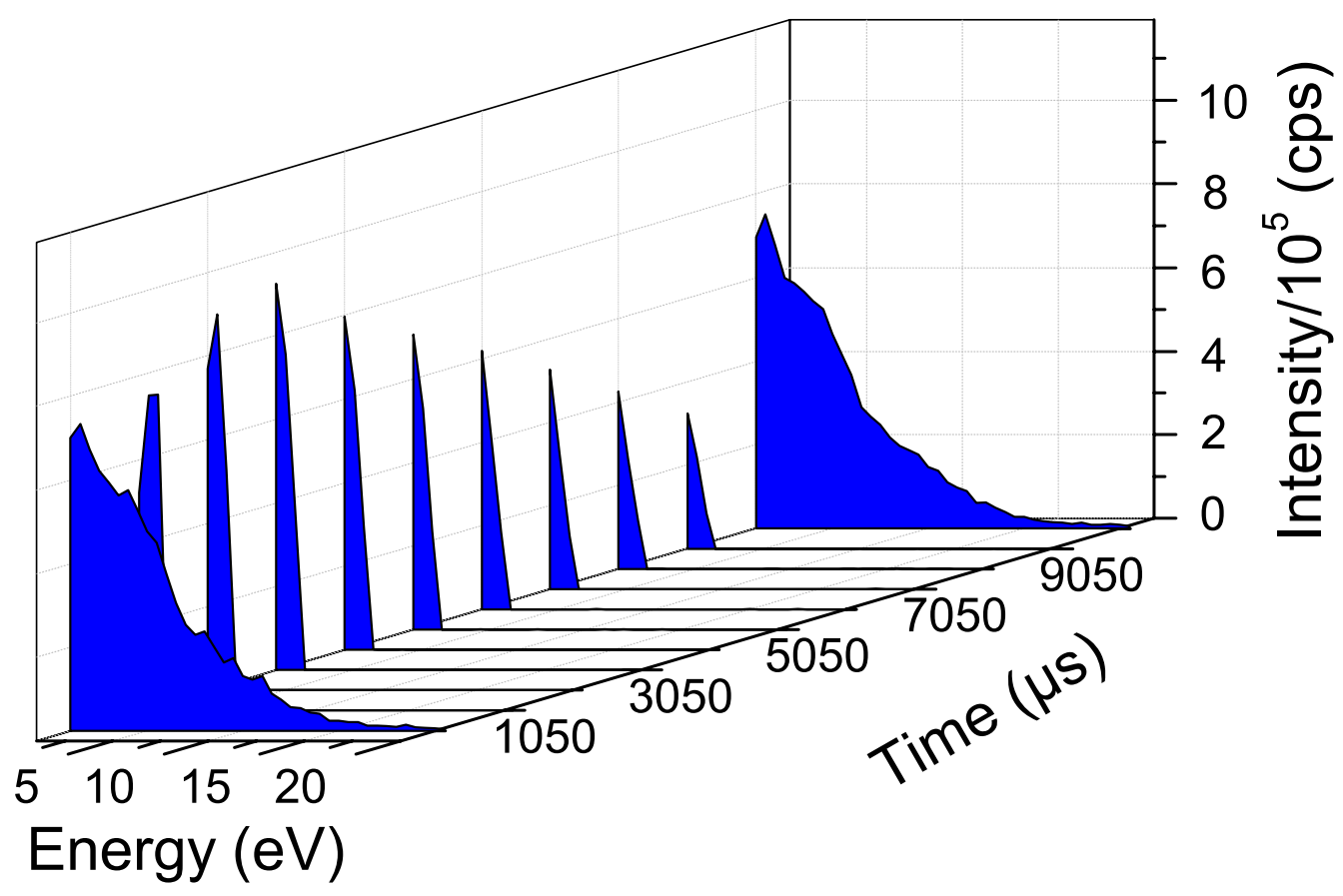

Figure 3. Time-resolved IEDFs of $\mathrm{Ag}^{+}$ions recorded from a discharge operated at $E_{p}=20 \mathrm{~mJ}$ and $f=100 \mathrm{~Hz}$. The presented data correspond to a pulsing period, i.e. the first IEDF was recorded 50 $\mu$ s from the pulse initiation and the last IEDF $50 \mu$ s after the onset of the next pulse. Only every fifth recorded IEDF is presented for clarity. 

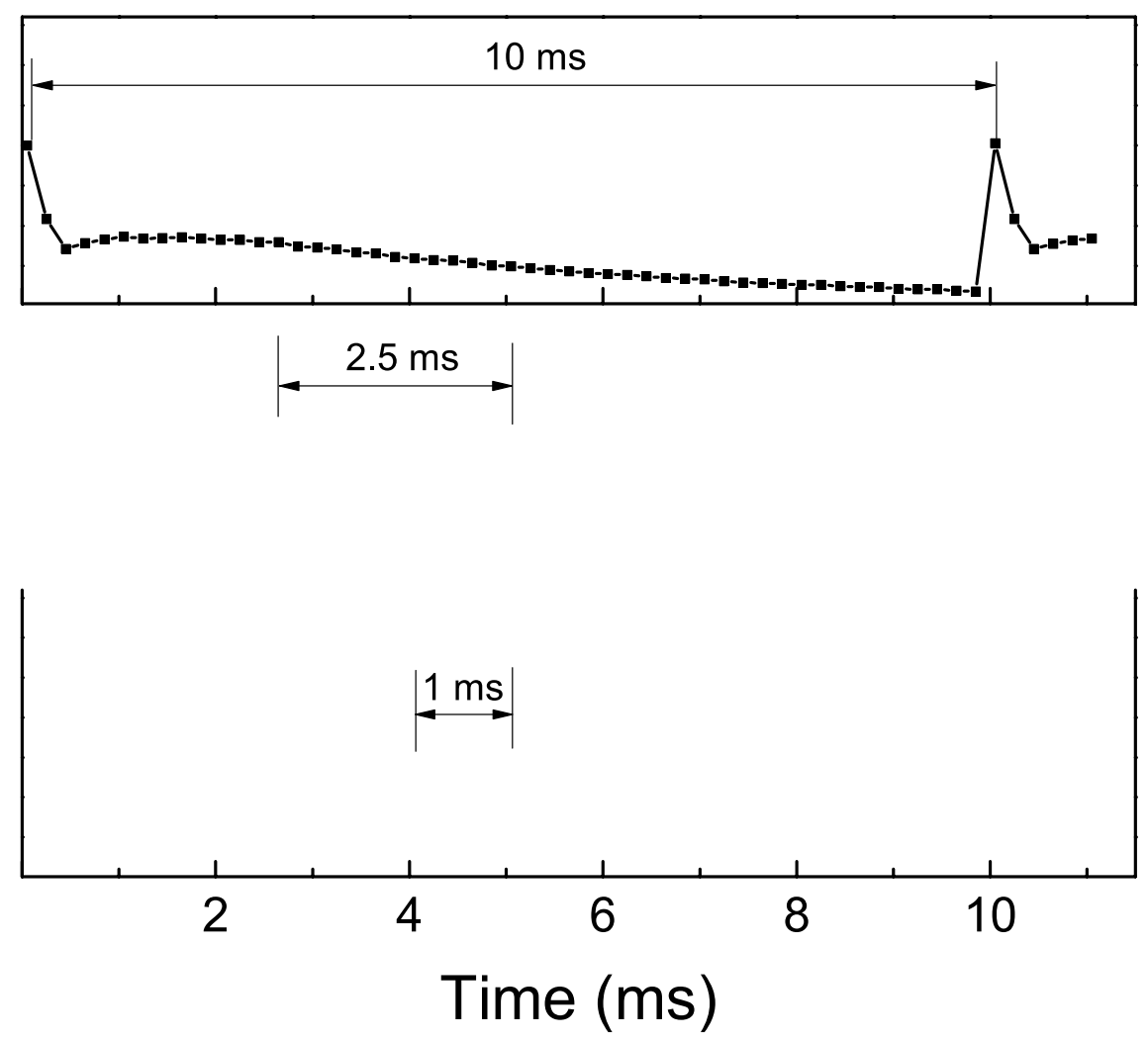

Figure 4. The time evolution of the integrated $\mathrm{Ag}^{+}$ion intensity at $E_{p}=20 \mathrm{~mJ}$ for $f=100,400$ and $1000 \mathrm{~Hz}$. 


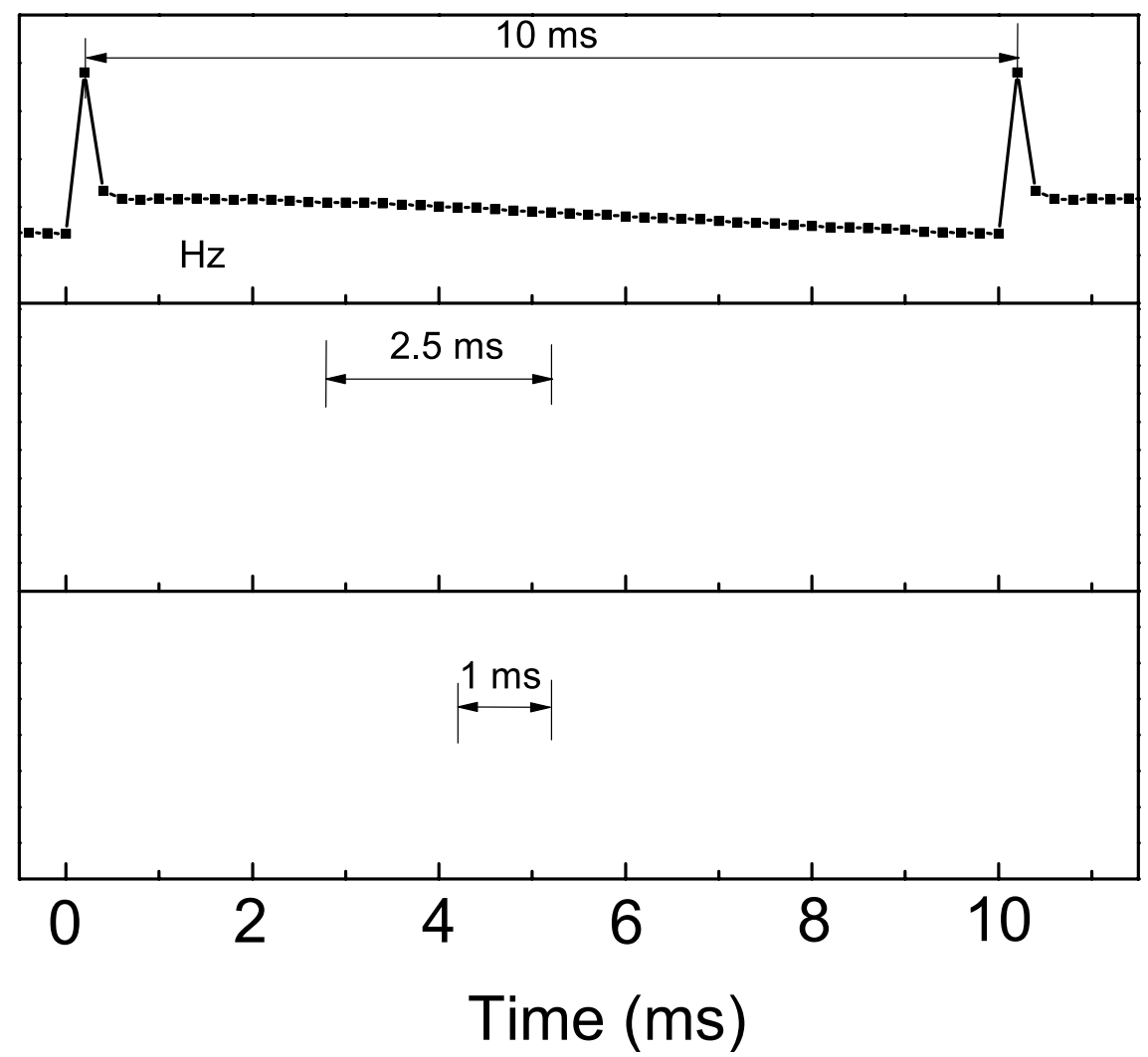

Figure 5. Simulated temporal profile of Ag neutral flux for $f=100,400$ and $1000 \mathrm{~Hz}$. 


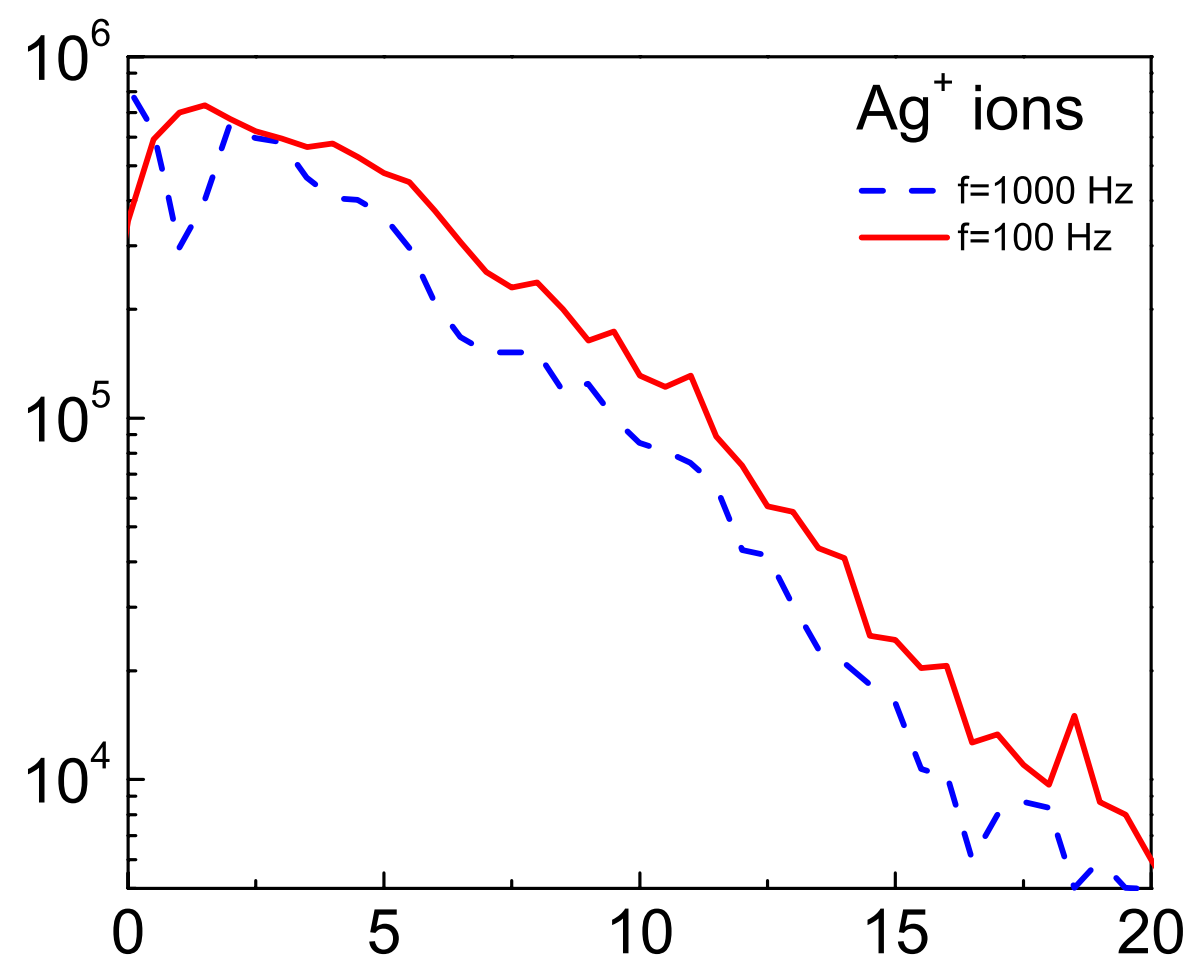

Figure 6. IEDFs of $\mathrm{Ag}^{+}$ions recorded for a discharge operating at $E_{p}=20 \mathrm{~mJ}$ and $f=100$ (solid line) and $1000 \mathrm{~Hz}$ (dashed line). The IEDFs were recorded $50 \mu$ s after the pulse initiation for a time interval of $200 \mu \mathrm{s}$. 


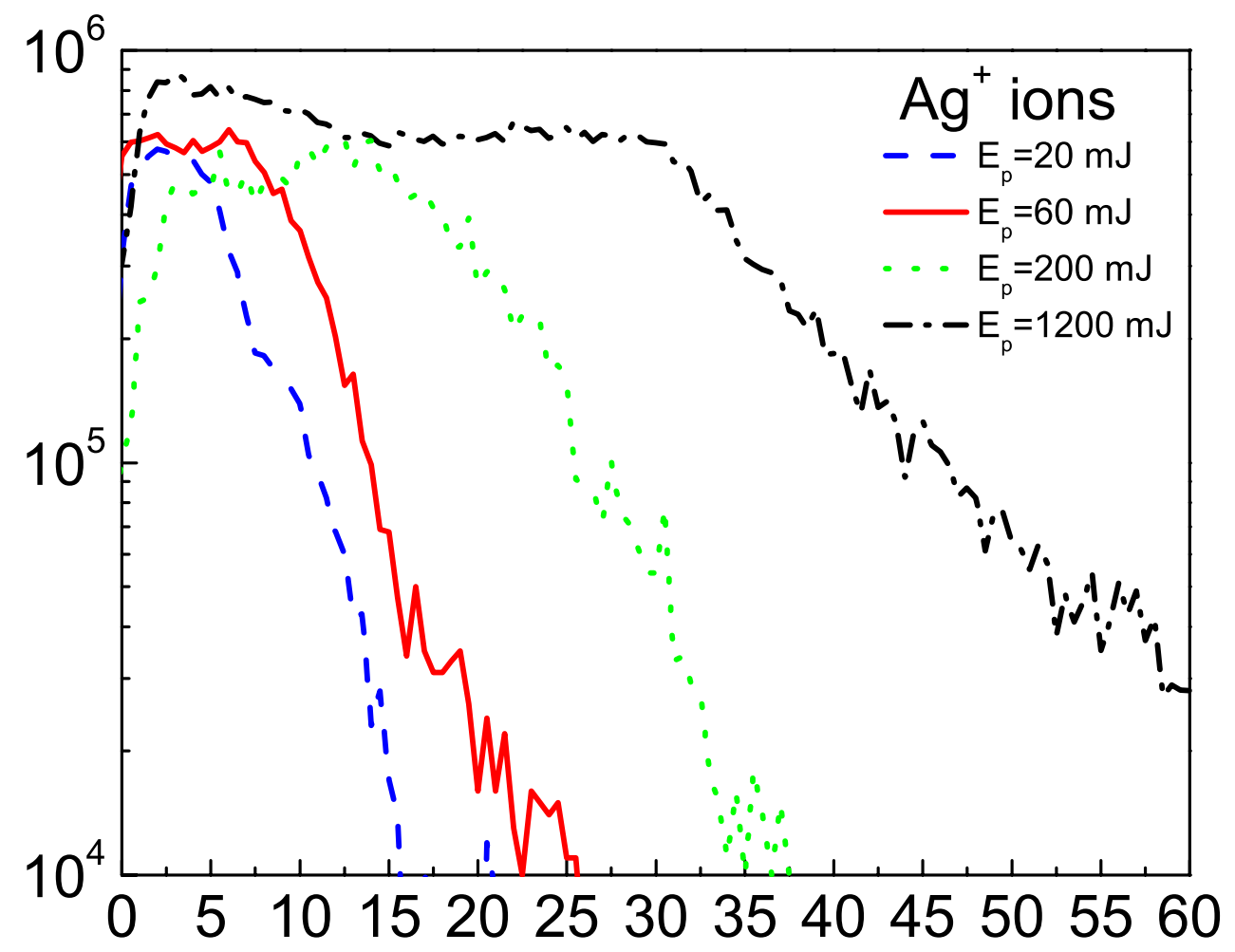

Figure 7. IEDFs of $\mathrm{Ag}^{+}$ions recorded for a discharge operating at $f=50 \mathrm{~Hz}$ and $E_{p}=20$ (dashed line), 60 (solid line), 200 (dotted line) and $1200 \mathrm{~mJ}$ (dashed-dotted line). The IEDFs were recorded $50 \mu$ s after the pulse initiation for a time interval of $200 \mu \mathrm{s}$. 


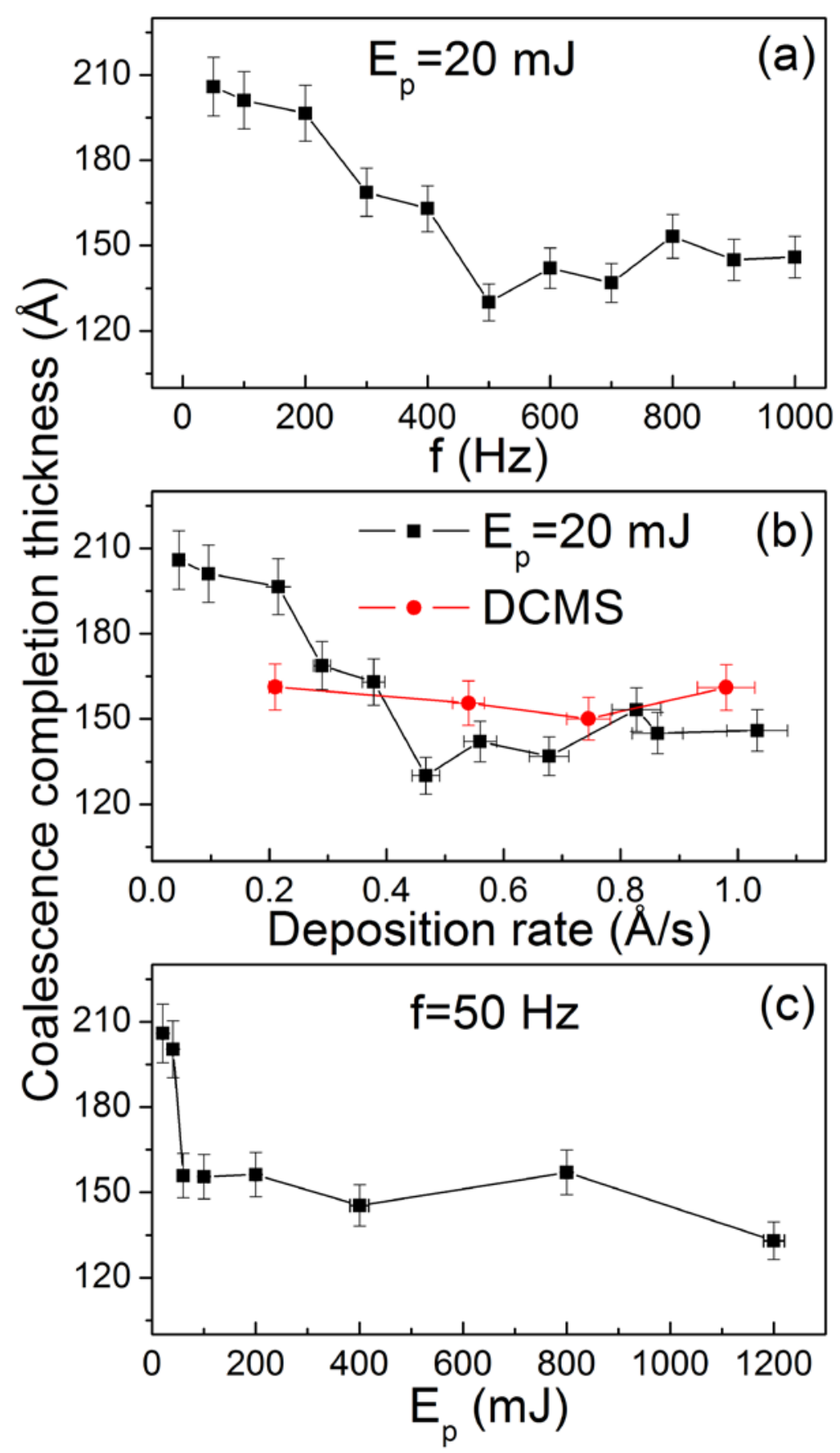

Figure 8. (a) Effect of pulsing frequency on the coalescence completion thickness at $E_{p}$ value of 20 mJ. (b) Dependence of coalescence completion thickness on deposition rate during pulsed ( $E_{p}=20 \mathrm{~mJ}, f=50 \mathrm{~Hz} / \mathrm{squares}$ ) and continuous (DCMS/circles) deposition. (c) Effect of pulse energy (at constant $f=50 \mathrm{~Hz}$ ) on the coalescence completion thickness. In all cases coalescence completion thickness values calculated from the intrinsic stress measurements are plotted. 


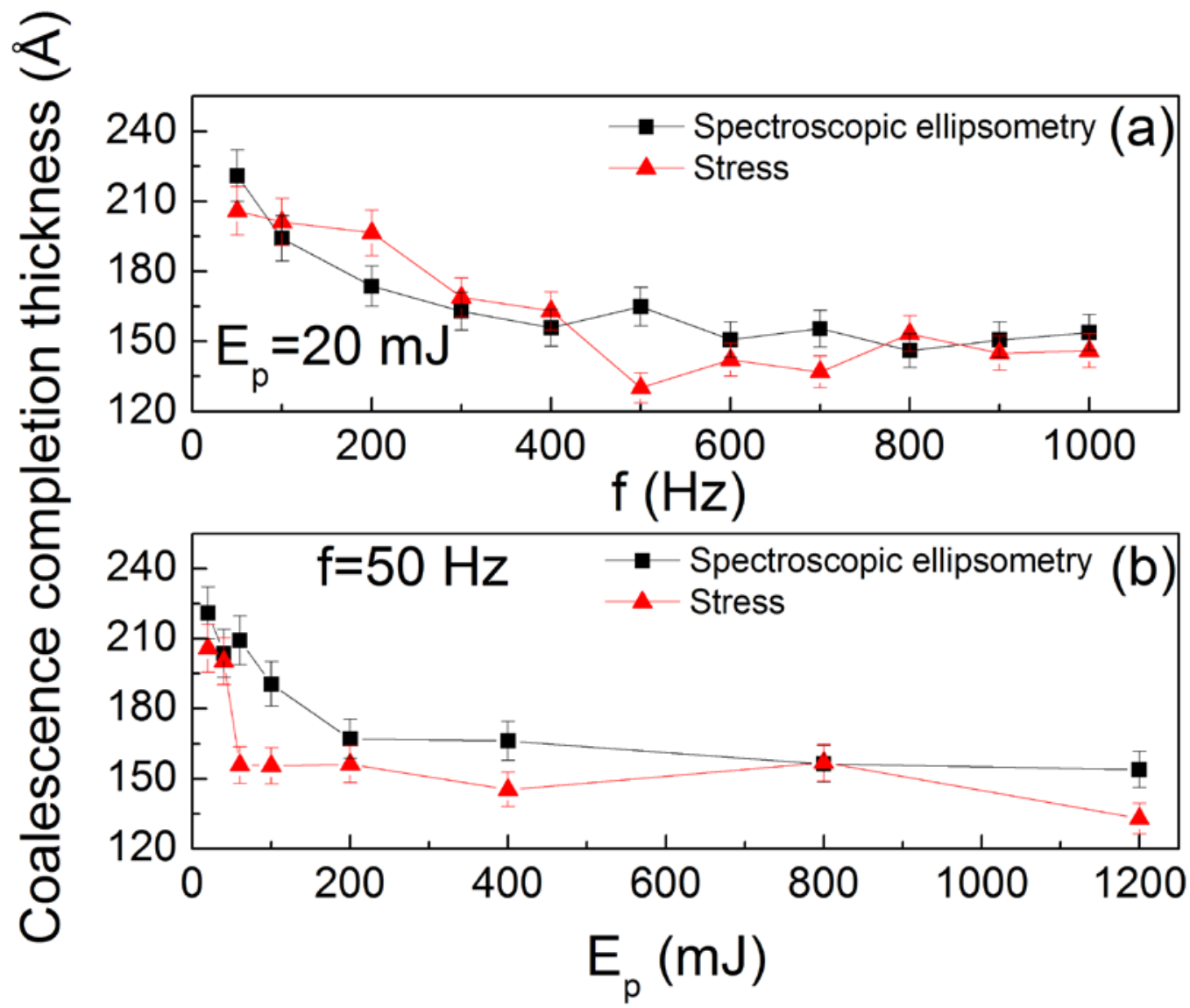

Figure 9. Coalescence completion thickness determined by spectroscopic ellipsometry (squares) as a function of (a) frequency at constant $E_{p}=20 \mathrm{~mJ}$ and (b) pulse power at constant $f=50 \mathrm{~Hz}$. The values obtained from the intrinsic stress measurements are also plotted for reference (circles). 


\section{Island radius, $R(\AA)$}

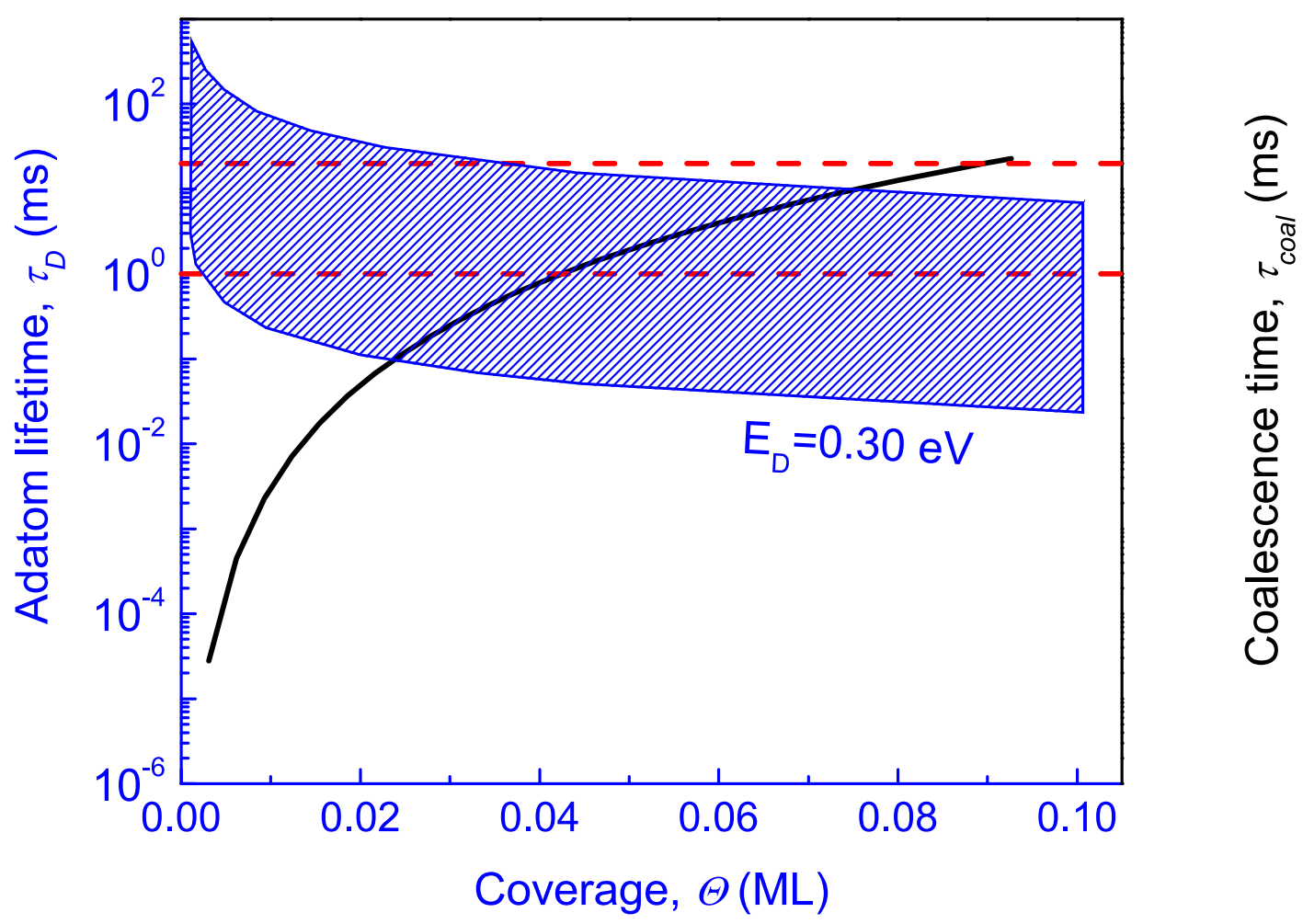

Figure 10. Adatom lifetime (shaded area, bottom-left axes) and coalescence time (continuous line, top-right axes) as a function of coverage and island radius, respectively. The bottom and top axes are not correlated. The boundaries of the shaded area correspond to adatom lifetimes calculated for diffusion barrier of 0.3 (bottom boundary) and $0.45 \mathrm{eV}$ (top boundary). The horizontal dashed lines indicate the extreme values of pulsing period of 20 and $1 \mathrm{~ms}$ (corresponding to 50 and 1000 $\mathrm{Hz}$ ) used in the experiments. 